\title{
Effects of Good Practices for Catch-Up Vaccinations: Assessment with a Quasi-Experimental Study in Democratic Republic of Congo
}

\author{
Yolaine Glèlè-Ahanhanzo', Alphonse Kpozèhouen', Collard Madika², Colette Azandjèmè3, \\ Chabi O. Alphonse Biaou ${ }^{1}$, Aristide Aplogan ${ }^{4}$ \\ ${ }^{1}$ Epidemiology and Biostatistics Department, Public Health Regional Institute, Ouidah, Benin \\ ${ }^{2}$ Ministry of Health, Kinshasa, Democratic Republic of the Congo \\ ${ }^{3}$ Health Promotion Department, Public Health Regional Institute, Ouidah, Benin \\ ${ }^{4}$ Agence de Médecine Préventive, Abidjan, Ivory Coast \\ Email: nyglele@yahoo.fr
}

How to cite this paper: Glèlè-Ahanhanzo, Y., Kpozèhouen, A., Madika, C., Azandjèmè, C., Biaou, C.O.A. and Aplogan, A. (2019) Effects of Good Practices for Catch-Up Vaccinations: Assessment with a Quasi-Experimental Study in Democratic Republic of Congo. Open Journal of Epidemiology, 9, 50-63.

https://doi.org/10.4236/ojepi.2019.91005

Received: December 5, 2018

Accepted: January 8, 2019

Published: January 11, 2019

Copyright $\odot 2019$ by author(s) and Scientific Research Publishing Inc. This work is licensed under the Creative Commons Attribution International License (CC BY 4.0).

http://creativecommons.org/licenses/by/4.0/

\begin{abstract}
Introduction: Despite the enormous resources deployed and the success achieved in vaccination, reducing the number of children who remain unvaccinated remains a major operational challenge in certain countries. In 2014, the Kalemie Expanded Program of Immunization outpost in the Democratic Republic of the Congo was faced with a high number of unvaccinated children. They instituted a "token" catch-up system based on a collaborative approach with community health workers. This study aims to document the effects of this strategy on vaccination coverage. Methods: This study was a comparative quasi-experimental test/control study. The "test" health area was Undugu where the catch-up strategy was implemented and the "control" health area was Kanunka. A vaccination coverage survey was conducted according to the WHO method in both health areas, examining children aged 12 to 23 months. The proportions were compared using a Pearson chi-square test and the odds ratios were estimated. Results: Vaccine coverage was significantly higher in the "test" health area for all the antigens. Full vaccination coverage was $69.4 \%$ in the "test" health area versus $31 \%$ in the "control" health area, with a 4.7 times greater chance of being fully vaccinated in the "test" health area (OR: 4.7; CI 95\% [3.1 - 7.2]; $\mathrm{p}=0.0001$ ). Conclusion: This study demonstrates the value of a strategy that can increase access to vaccination and use of vaccination services, leading to a reduction in inequality in this area. However, this is linked to adapting the collaborative model on
\end{abstract}


which it is based. This must be taken into consideration in plans to reproduce the findings.

\section{Keywords}

Immunisation Programs, Vaccination Coverage, Community Participation, Non-Randomized Controlled Trials as Topic

\section{Introduction}

The many benefits of vaccination, presented as one of the most cost-effective interventions in healthcare, have been well documented [1] [2] [3]. Despite the progress observed in vaccination coverage in most countries, access to vaccination continues to be insufficient for many children around the world, particularly in developing countries. Looking beyond the overall vaccination coverage figures, relatively large disparities can be seen, linked to a number of factors. According to the report published by the WHO in 2013 "nearly 22 million infants missed out on the required three doses of diphtheria-tetanus-pertussiscontaining vaccines" (DTP3), many of them living in the world s poorest countries [4] [5]. The current global vaccination table shows the need to increase efforts to improve a system that, although relatively functional in most countries, must aim to reduce the number of children who are not reached, or do not complete vaccination schedules, to as few as possible in order to reduce vaccination inequality [6] [7]. Every child has the right to be vaccinated and a number of strategies have already been implemented, primarily in developing countries, in particular the Reaching Every District (RED), Reaching Every Community (REC) and now the Reaching Every Last Child (RELC) approaches, as well as additional vaccination strategies of various forms such as National Vaccination Days (NVDs), Local Vaccination Days (LVDs), etc. [8]. The results obtained remain particularly mixed, with many variables depending on the context and socio-cultural circumstances limiting access to vaccination [9] [10]. These are particularly apparent in large countries with socio-economic disparities and especially unusual political circumstances, such as the Democratic Republic of the Congo (DRC). Despite laws on child protection and requirements for child vaccination, these services are not widely available and a large number of infants are not vaccinated [11] [12]. Given the importance of vaccination, and in light of the delay in reaching the MDGs, the WHO and member countries have implemented several approaches aiming to shorten the time taken to achieve the MDGs, as stipulated in the Global Vaccine Action Plan 2012-2020 [3]. Most of these strategies are based on full community participation via multiple networks to reach the targets. In addition to the main components of the RED approach, this principle was applied through the "token" catch-up technique in certain health areas in the field of vaccination in Kaliemie province. This initiative was a way to improve the usual methods for reaching unvaccinated targets. It was in- 
tended to be an operational response to the observation made in the anthropological study of vaccination refusal and resistance in Katanga. This anthropological study was conducted with support from UNICEF and stresses that innovative vaccination promotion strategies be implemented that must take all behavioural factors into account, and which are supported by beliefs, if populations are to be convinced of the benefits and safety of vaccines [13]. This study aims to document the effects of this catch-up technique on child vaccination coverage, seeking to provide arguments for, or against, the use of best practices, as encouraged in the Global Vaccine Action Plan 2011-2020.

\section{Methods}

\subsection{Framework of the Study}

This study was conducted in the Undugu health area, the intervention health area (IHA) in the Nyema health zone, and the Kanunka control health area (CHA) in the Mulongo health zone. Both areas have received support from the Bill \& Melinda Gates Foundation (BMGF). The Undugu health area is one of 21 health areas in the Nyemba health zone in the Tanganyika provincial health district (PHD). It is an urban health area, with a rural zone that in 2014 was home to 55,683 people. The Kanunka health area, one of 24 health areas in the $\mathrm{Mu}$ longo health zone, in the Haut-Lomami provincial health district. The total population in 2015 was 9235 inhabitants (extrapolation from 2014 data). This health area has a health centre as well as a health development committee that trains community health workers. The HA, which has not used tokens, was selected as the control zone based on the following criteria; belonging to another health zone far from the Nyemba health zone $(577 \mathrm{~km})$ to avoid any ripple effect or influence, the absence of any other interventions of the same type or with the same objectives in the control zone, a similar population in the two HAs, similar administrative vaccination coverage in 2013 for full vaccination in children aged one year (Undugu 50\% and Kanunka 52\%) and similarity in the organisation and delivery of vaccination services other than in the intervention, this being the child catch-up technique for children that are insufficiently vaccinated using the token method in the test zone.

\subsection{Description of the Intervention}

The intervention used the child vaccination catch-up technique involving a card called a "token" used by three parties: the health centre's operations unit, community health workers and the child's parents/guardian. It is based on identifying children that were absent from a vaccination session or that had never been to vaccination services. This identification is done by both the nurse at the health centre and by the community health worker within the community. The token serves to identify vaccination catch-up targets, along with all the services that the target has missed, and thus the antigens to be caught-up with, as well as a tool for monitoring the intervention, ensuring that all actions by the three par- 
ties can be tracked. In this method, the vaccination catch-up begins immediately following a vaccination session. This session is organised with the community health worker responsible for the site present.

Following the vaccination, the vaccinator identifies any children that did not attend the session and draws up a list with the addresses and the antigens they missed. Based on this list, the vaccinator groups the children in question according to location within villages/blocks (village, neighbourhood, block or street). Each group or block is assigned to the location's community health worker. Once this grouping has been carried out, tokens are filled in, with one token produced for each child. This document includes the child's name, age (in months), vaccination register number, the antigens requiring catch-up and the date (day) the mother/guardian arrived at the vaccination location with the child. It should be noted that a target child could miss two, three or even more antigens from a single vaccination session. All antigens that are missing for a child are listed on a single token, stating the day of the meeting. After this token is filled in, the vaccinator identifies the appropriate community health worker and presents them with the tokens for their activity area. At this stage, the vaccinator explains and reminds the community health worker of the different antigens that each child needs to receive and the diseases they protect the child from. If the community health worker is present, they are given the token at the end of the vaccination session, or if not present, following the vaccination session but as soon as possible before the date of the meeting.

Each community health worker with children identified as requiring catch-up must, after taking the tokens from the vaccinator, in turn deliver the tokens to the parents/guardians of the children concerned. The handing over of the token is an opportunity to raise awareness and identify the reasons the child was not vaccinated, which will be reported to the nurse so that appropriate strategies can be identified. Here, the community health worker must also demonstrate the benefits to the child, parents, family and overall community of the child being vaccinated, as well as the risks they face if the child is not vaccinated. At this point, the community health worker records the name of each child requiring catch-up in their notebook, before leaving the token with the parents. Each parent must sign, or initial, the name of their child in the community health worker's notebook. Finally, the community health worker will write their name on the back of the token given to the parent/guardian. During the home visit, the community health worker may identify another child that has never been vaccinated. This child will also receive a token, collected from the nurse responsible for this at the centre or a colleague.

The child's parent/guardian must bring the token and present it to the vaccinator at the following session, which is the meeting detailed on the token. The vaccinator will present the token to the head nurse at the health centre to identify the children that did, and did not, come to the meeting. Next, the nurse at the centre will archive the tokens by month, by village/neighbourhood/street and by vaccination location. For parents that have not brought their children, despite 
the visit from the community health worker, the health centre team must decide on additional strategies to persuade them to do so.

This technique requires good organisation and full compliance by all those involved; it is only successful when the health area has dynamic community health workers, who are motivated and properly involved in all five areas of the Reach Each Zone/Reach Each District approach. To maintain the motivation and interest of the community health workers, who are volunteers, incentives were introduced. Two notable examples are an exemption from certain medical fees for themselves, their spouse and children, and giving priority to these community health workers when selecting workers for paid positions in mass vaccination campaigns, etc.

\subsection{Study Type, Population and Sampling}

This was a comparative quasi-experimental study, where vaccination coverage was compared using data from surveys conducted in the Undugu health area, which used tokens (intervention health area- IHA) and vaccination coverage in the Kanunka health area, which did not use tokens, in 2014 (control health area-CHA). It examined mainly parent/guardian couples with children aged 12 to 23 months in the two health areas, children aged 12 to 23 months in the two health areas being studied, and secondarily the community health workers, nurses at the health centres and contact people. The survey was conducted in July 2015.

The sampling method used was the two-stage cluster sampling method recommended by the WHO. The sampling frames were created from the list of villages in each health area. The target population in each health area (children aged 12 to 23 months) was identified by extrapolating data from the 2014 population and the villages in the two health areas. The sample size was calculated based on the expected proportion of target children compared with the number of fully immunised children (50\%) using the Schwartz formula with a $1 \%$ accuracy and cluster effect of $k=2$. The sample size calculated was 192 . The sample size per cluster was $192 / 30=6.40$ per cluster, rounded to 7 . Thus, the actual sample size used was 210 couples in each health area. From the list of villages in each HA, the 30 clusters were selected using the cumulative population method. Households were selected at random, then the targets were selected at random from the households, with at most one target per household [14].

\subsection{Techniques, Data Collection Tools and Variables}

We conducted a structured interview, using a questionnaire, of the parents/guardians of the child aged 12 to 23 months, as well as looking for the vaccine scar and inspecting the vaccination card. To collect information from service providers (nurse at the health centre, district management team, community health workers), we used an observation grid. The inspection of the document was conducted using a processing form; we also conducted interviews us- 
ing a structured questionnaire. The questionnaire was pre-tested in Kalemie Centre, in the Lubuye health area, outside of the intervention and control zones. We were interested in:

$\checkmark$ Child's age (in months): [12 to 15], [15 to 17], [17 to 21], [21 to 23]

$\checkmark$ Child's gender

$\checkmark$ Vaccination status of the child for the BCG, DTP-HepB-Hib3, Pneumo-3, OPV-3, VAR and yellow fever antigens: Vaccinated or Not vaccinated

$\checkmark$ Fully immunised child status (FIC): Yes or No

$\checkmark$ Age of parent/guardian interviewed (in years): [15 - 19], [20 - 29], [30 - 39], [40 or over]

$\checkmark$ Sex of parent/guardian interviewed

$\checkmark$ Relationship of parent/guardian interviewed with the child: Father, mother, guardian

$\checkmark$ Marital status of parent/guardian interviewed: In a relationship/single

$\checkmark$ Level of education of parent/guardian interviewed: "Secondary education or higher", "primary education", "no education"

$\checkmark$ Occupation of parent/guardian interviewed: Farmer, housewife, trader, employee

$\checkmark$ Religion of parents: Catholic, Protestant, Muslim, Kimbanguist, Other

$\checkmark$ Reasons for not being vaccinated

Vaccination coverage (VC) was calculated for each antigen based on vaccination cards in each HA, using the following formula:

$\mathrm{VC}($ in $\%)=($ Number of children surveyed that had received the antigen/total number of children surveyed) $\times 100$.

\subsection{Data Processing and Analysis}

The processing and analysis of the date was carried out using SPSS-20 software. We used conventional descriptive statistics with qualitative variables presented proportionally. The comparisons were made using the chi square test with a lower threshold of 0.05 . We estimated odds ratios (ORs) followed by the confidence interval of $95 \%$ (CI 95\%).

\subsection{Ethical Considerations}

This study complied with international ethical standards. The aims of the study were explained to participants, who were also reassured of the confidentiality of what was discussed. An information sheet was distributed, and the questionnaire was only administered to mothers or guardians of children after obtaining their free and informed consent. We also obtained authorisation from all the administrative and health authorities concerned before beginning the survey.

\section{Results}

\subsection{Description of the Sample}

Overall, the socio-demographic characteristics were comparable for respondents 
in the two study areas (Table 1). There was no statistically significant difference between the populations of the two health areas with respect to the age of the parent/guardian interviewed $(\mathrm{p}=0.54)$; level of education of the parent/guardian interviewed $(p=0.67)$; the religion of the parents $(p=0.21)$; the marital status of the parents $(p=0.34)$ or the sex of the child $(p=0.845)$. Looking at the distribution of the age of the children, a statistically significant different was observed $(p=0.047)$.

\subsection{Comparison of Vaccination Coverage in Children Aged 12 to 23 Months}

The data collected from vaccination cards shows the percentage of fully immunised children (FIC) at $69.4 \%(\mathrm{n}=146)$ in the health area with tokens (IHA) compared with $31 \%(\mathrm{n}=65)$ in the health area without tokens $(\mathrm{CHA})$. There was 4.7 times the chance of a child being fully vaccinated if they lived in a health area using tokens compared to a child living in a health area not using tokens (OR: 4.7; CI 95\%: [3.1 - 7.2]; $\mathrm{p}=0.0001$ ). The highest vaccination coverage in the IHA was the BCG (87.14\%), followed by DTP-HepB-Hib-3 and OPV-3 with $83.81 \%$ per antigen. Yellow fever was the antigen with the lowest coverage (75\%) during the period considered. The same observation was made in the CHA, however with different proportions: BCG (68\%), DTP-HepB-Hib-3 (63\%) and OPV-3 (61\%). Vaccination coverage by antigen in the IHA was significantly higher than that found in the CHA (Table 2).

\subsection{Reasons for Not Being Vaccinated in the Case Health Area}

Only parents of children who were not fully immunised were asked this question, which was a total of 64 parents (30.6\%). Of these, $81 \%$ cited the mother not being available (travel, illness); the child being ill (70\%); the parents being busy with other activities (60\%); in 50\% of cases, it was attributable to rumours about vaccines and vaccination. We observed that $38.1 \%$ were not aware of the need for vaccination, $33.3 \%$ did not know about needing to return to the health centre to finish the vaccination of their children (taking second or third doses in a schedule); $27.6 \%$ said that the staff at the facility were too busy, and $30.8 \%$ of the parents were worried about adverse events following immunisation (AEFI).

\section{Discussion}

\subsection{Comparison of the Samples in IHA and CHA}

This comparative quasi-experimental study was conducted in two health areas: the intervention health area of Undugu which tested a collaborative catch-up method for children who were not or insufficiently vaccinated, with the aim of helping increase vaccination coverage, and the control health area of Kanunka, which did not use the catch-up method.

In terms of socio-demographic characteristics, the two populations are comparable in most areas: The age of the parents $(\mathrm{p}=0.54)$, the gender of the 
Table 1. Distribution of the general characteristics of the interviewees by health area.

\begin{tabular}{|c|c|c|c|c|c|}
\hline \multirow{2}{*}{ Variable } & \multicolumn{2}{|c|}{$\operatorname{IHA}(\mathrm{n}=210)$} & \multicolumn{2}{|c|}{$\mathrm{CHA}(\mathrm{n}=210)$} & \multirow{2}{*}{$\begin{array}{c}\mathrm{p} \\
\text { value }\end{array}$} \\
\hline & Number & $\%$ & Number & $\%$ & \\
\hline Age of parent/guardian interviewed (in years) & & & & & 0.54 \\
\hline$[15-19]$ & 37 & 17.62 & 27 & 12.86 & \\
\hline$[20-29]$ & 115 & 54.76 & 117 & 55.71 & \\
\hline$[30-39]$ & 51 & 24.29 & 59 & 28.10 & \\
\hline [40 or over] & 7 & 3.33 & 7 & 3.33 & \\
\hline Sex of parent/guardian interviewed & & & & & 0.07 \\
\hline Male & 12 & 5.71 & 22 & 10.48 & \\
\hline Female & 198 & 94.29 & 188 & 89.52 & \\
\hline Level of education of parent/guardian interviewed & & & & & 0.67 \\
\hline Secondary or higher & 23 & 10.95 & 29 & 13.81 & \\
\hline Primary & 169 & 80.48 & 163 & 77.62 & \\
\hline No education & 18 & 8.57 & 18 & 8.57 & \\
\hline Occupation of parent/guardian interviewed & & & & & $<0.01$ \\
\hline Farmer & 29 & 13.81 & 119 & 56.67 & \\
\hline Housewife & 27 & 12.86 & 70 & 33.33 & \\
\hline Trader & 123 & 58.57 & 15 & 7.14 & \\
\hline Employee & 31 & 14.76 & 6 & 2.86 & \\
\hline Religion of parents & & & & & 0.21 \\
\hline Catholic & 57 & 27.14 & 55 & 26.19 & \\
\hline Protestant & 128 & 60.95 & 129 & 61.43 & \\
\hline Muslim & 13 & 6.19 & 6 & 2.86 & \\
\hline Kimbanguist & 3 & 1.43 & 2 & 0.95 & \\
\hline Not specified & 9 & 4.29 & 18 & 8.57 & \\
\hline Marital status & & & & & 0.34 \\
\hline In a relationship & 193 & 91.90 & 198 & 94.29 & \\
\hline Single & 17 & 8.10 & 12 & 5.71 & \\
\hline Link between parents and children & & & & & 0.12 \\
\hline Father & 12 & 5.71 & 20 & 9.52 & \\
\hline Mother & 198 & 94.29 & 188 & 89.52 & \\
\hline Guardian & 0 & 0.00 & 2 & 0.95 & \\
\hline Gender of children & & & & & 0.845 \\
\hline Male & 96 & 45.71 & 94 & 44.76 & \\
\hline Female & 114 & 54.29 & 116 & 55.24 & \\
\hline Age of children (in months) & & & & & 0.047 \\
\hline$[12-15]$ & 70 & 33.33 & 74 & 35.24 & \\
\hline$[15-17]$ & 52 & 24.76 & 73 & 34.76 & \\
\hline$[17-21]$ & 46 & 21.90 & 35 & 16.67 & \\
\hline$[21-23]$ & 42 & 20.00 & 28 & 13.33 & \\
\hline
\end{tabular}


Table 2. Vaccination coverage by antigen and by health area.

\begin{tabular}{cccccc}
\hline \multirow{2}{*}{ Variable } & \multicolumn{2}{c}{ IHA $(\mathrm{n}=210)$} & \multicolumn{2}{c}{ CHA $(\mathrm{n}=210)$} & \multirow{2}{*}{ p value } \\
\cline { 2 - 5 } & Number & VC $(\%)$ & Number & VC $(\%)$ & \\
\hline BCG & 183 & 87.14 & 143 & 68.10 & $<0.001$ \\
DTP-HepB-Hib-3 & 176 & 83.81 & 132 & 62.86 & $<0.001$ \\
OPV-3 & 176 & 83.81 & 128 & 60.95 & $<0.001$ \\
VAR & 162 & 77.14 & 107 & 50.95 & $<0.001$ \\
Yellow fever & 158 & 75.24 & 105 & 50.00 & $<0.001$ \\
FIC & 145 & 69.05 & 67 & 31.90 & $<0.001$ \\
\hline
\end{tabular}

parents of the children $(p=0.07)$, the levels of education of the parents at both sites $(\mathrm{p}=0.67)$, the religion of the parents $(\mathrm{p}=0.21)$, their marital status $(\mathrm{p}=$ 0.34 ), the relationship between the parents/guardians and the target children ( $p$ $=0.12)$ and the gender of the children aged 12 to 23 months $(p=0.845)$. A difference was observed when looking at the occupation of the parents $(p=0.0001)$ and the age of the children $(p=0.047)$. The difference between the two populations in these two variables does not however significantly influence the comparison between the two health areas studied. With respect to the occupation of the parents; farmers and traders are professional groups that are particularly active, however they have well-defined working hours in general that do not limit access to vaccination services in any way, neither for the parents themselves, nor for those involved in the healthcare system. The difference between the results of two with respect to the age of the children should also not influence the results, as for vaccination the children are passive participants and it is the parents that decide.

\subsection{Comparison of the Vaccination Coverage in IHA and CHA}

The percentage of fully immunised children (FIC) in the intervention health area is close to the figures reported in Cameroon in 2014, in a study conducted in the district of Djoungolo in an urban environment, on the factors behind children not being fully immunised [15]. However, this vaccination coverage was higher than in the control health area and even those found in national statistics from 2011 in Ethiopia, which reported the percentage of fully immunised children at $25 \%$ [16]. This difference could be explained in part by the fact that, according to the authors, low vaccination coverage in Ethiopia was linked to the absence of appropriate strategies to improve vaccination coverage, especially in rural populations and populations with major issues concerning geographical accessibility. Moreover, this Ethiopian study was based on the National Demographic and Health Survey (EDS) and thus included all environments, while our study was a local study representative of a specific context.

Comparing vaccination coverage in the two health areas included in our study, there was a significant difference in the vaccination coverage of all anti- 
gens and the percentage of fully immunised children. This shows the positive effects of the catch-up method in increasing vaccination coverage in the IHA compared with the CHA, it being an effective approach that can contribute to reaching the targets set by the WHO. In terms of fully immunised children (FIC), we observed that children in the IHA were five times more likely to be fully immunised than those in the CHA, a major difference between the IHA and the CHA. These results would argue in favour of interventions to improve vaccination performance through community health workers and community participation in general. In Kenya, between 2013 and 2015, the contribution of community health workers was assessed in rural environments, with a before-and-after comparison between intervention and control areas. The results of this study correspond with ours, although with a much smaller increase of $10 \%$ in the percentage of fully immunised children in the intervention area compared to the control area and a likelihood of being fully vaccinated 2.5 times higher in the intervention area compared with the control area [17]. The differences between our results and this study may be due to the initial conditions in the intervention areas in the Kenyan study. The baseline studies actually show the percentage of fully immunised children in the two areas at around $85 \%$, whilst in our study, the percentage was around $50 \%$. The principle of diminishing marginal returns could certainly be part of the reason for the positive effects in the Kenyan study being less than in our study. The authors of a study in India in 2010 stress that although there were not many studies comparing the effectiveness of interventions involving community health workers and other vaccination interventions, it is certain that "CHOs contribute to improving vaccination programmes in a range of ways" [18]. Similarly, in Myanmar (formerly Burma) in 2016, an experiment by the Ministry of Health confirmed the importance of community health workers in reducing the gap in access to primary healthcare for populations that are disadvantaged and particularly difficult to access [19].

\subsection{Reasons for Not Being Vaccinated in the IHA}

Despite the effectiveness of the intervention in the IHA, there were children that were not vaccinated. All of the reasons mentioned by the parents, in particular the mothers not being available, rumours about vaccines and vaccination, ignorance about the need for vaccination and the vaccination series to be completed, and fears about AEFI, are strongly correlated with summaries of reasons from literature on reasons for children not being vaccinated in medium- and low-income countries [20]. The reasons mentioned in our study by the 64 parents/guardians for not having their children vaccinated were primarily the mothers not being available and parents being occupied with other activities in general. This was probably the most common reason, as vaccination is considered the responsibility of the mother who, in most cases, was not at the home, and was looking for ways to contribute to the survival of the family, as were their husbands. Children being ill as a reason for not vaccinating children aged 12 to 
23 months was mentioned by $70 \%$ of parents. Very often, the parents did not agree to have their sick child vaccinated despite the child already receiving injections to treat the current illness. This is often due to the belief that the vaccination will be an inconvenience, and also likely an expression of ignorance of the need for vaccination; the case in $38.1 \%$ of parents. Similar to the other reasons mentioned for not having their children vaccinated, such as rumours about the vaccine and vaccination, also mentioned in the literature [21], and ignorance of the need to return to complete the vaccination of their children. These factors confirm that the level of education of the parent(s)/guardian(s), and more often the mother, play a key role in the vaccination of children [22] [23]. Communication is therefore essential to raise awareness among parents about vaccination. Hence the importance of increasing the skills of the community health workers as part of the intervention, as they are the first point of contact for parents. To supervise the health workers and to make the most of any opportunity when the parents are in contact with healthcare services to raise awareness about vaccination and recommend vaccines to other people, with a special focus on mothers [24] [25] [26]. These assorted contact points will also reassure mothers who have concerns about adverse events following immunisation (AEFI).

The reorganization of vaccination services for greater effectiveness by being more in-line with the activities of the parents is also a necessity, as many healthcare facilities fail to manage them efficiently for a range of reasons, often including a lack of expertise or people. As many as one in three parents stated that their children were not vaccinated because staff at the facility were too busy. In the context, given that parents are generally occupied with other activities in the morning, the teams arrange vaccination sessions in the afternoon. However, they have little time because staff numbers are insufficient to cover all the activities before nightfall. Advocacy in favour of more human resources for health centres is needed in order to cover the vaccination needs of the population. This is because, as has been demonstrated in multiple works, in vaccination, the density of nursing staff is associated with infant vaccination coverage in developing countries [27] and a higher density of human resources increases the availability of services in both time and location, which increases the likelihood of achieving infant vaccination targets [28].

\subsection{Limitations of the Study}

The aim of this study was to investigate the effects of a model of the catch-up technique on child vaccination coverage, seeking to provide arguments for, or against, the use of these best practices. The findings suggest that we achieved our objectives. However, the study may be limited in its scope. As a quasi-experimental study, it was not possible to guarantee complete control of external factors. Ideally, there should be a second comparison with a baseline in both of the study health areas. Without this baseline, we chose health areas that were similar in context, in the performance of child immunisation program and in the funding of the immunisation program, seeking to limit bias. The similari- 
ty of our samples in the IHA and CHA confirmed the comparability of our populations.

\section{Conclusion}

This study has allowed us to highlight the positive effect of a catch-up method for children that are either not vaccinated, or insufficiently vaccinated, based on a collaborative model with the community, and confirms the hypothesis behind this work, that the use of this method would be an effective contribution to increasing vaccination coverage. However, this strategy should be expanded to increase effectiveness. For this, existing reasons for children not being vaccinated must be accounted for and the incentive system for community health workers must be contextualised.

\section{Conflicts of Interest}

The authors declare no conflicts of interest regarding the publication of this paper.

\section{Availability of Data and Materials}

Data collection tools, dataset used and analysed during the current study are available from the first author on reasonable request.

\section{References}

[1] Greenwood, B. (2014) The Contribution of Vaccination to Global Health: Past, Present and Future. Philosophical Transactions of the Royal Society of London Series B, Biological Sciences, 369, Article ID: 20130433. https://doi.org/10.1098/rstb.2013.0433

[2] John, T.J. and Vashishtha, V.M. (2013) Eradicating Poliomyelitis: India's Journey from Hyperendemic to Polio-Free Status. The Indian Journal of Medical Research, 137, 881-894.

[3] Organisation Mondiale de la Santé (2013) Plan d'action mondial pour les vaccins 2011-2020. OMS, Genève.

[4] WHO (2013) Statistiques sanitaires mondiales. World Health Organisation, Geneva.

[5] Harris, J.B., Gacic-Dobo, M., Eggers, R., Brown, D.W. and Sodha, S.V. (2014) Global Routine Vaccination Coverage, 2013. MMWR Morbidity and Mortality Weekly Report, 63, 1055-1058.

[6] Hinman, A.R. and McKinlay, M.A. (2015) Immunization Equity. American Journal of Preventive Medicine, 49, S399-S405. https://doi.org/10.1016/j.amepre.2015.04.018

[7] Casey, R.M., Hampton, L.M., Anya, B.M., Gacic-Dobo, M., Diallo, M.S. and Wallace, A.S. (2017) State of Equity: Childhood Immunization in the World Health Organization African Region. The Pan African Medical Journal, 27, 5. https://doi.org/10.11604/pamj.supp.2017.27.3.12114

[8] Sodha, S.V. and Dietz, V. (2015) Strengthening Routine Immunization Systems to Improve Global Vaccination Coverage. British Medical Bulletin, 113, 5-14. https://doi.org/10.1093/bmb/ldv001 
[9] Minetti, A., Kagoli, M., Katsulukuta, A., Huerga, H., Featherstone, A., Chiotcha, H., et al. (2013) Lessons and Challenges for Measles Control from Unexpected Large Outbreak, Malawi. Emerging Infectious Diseases, 19, 202-209. https://doi.org/10.3201/eid1902.120301

[10] Machingaidze, S., Wiysonge, C.S. and Hussey, G.D. (2013) Strengthening the Expanded Programme on Immunization in Africa: Looking beyond 2015. PLoS Medicine, 10, e1001405. https://doi.org/10.1371/journal.pmed.1001405

[11] UNICEF (2017) Immunization Coverage by Antigen (Country, Regional, and Global Trends). UNICEF, New York.

[12] Ministère du Plan et Suivi de la Mise en œuvre de la Révolution de la Modernité-MPSMRM/Congo, Ministère de la Santé Publique-MSP/Congo (2014) ICF International. République Démocratique du Congo Enquête Démographique et de Santé (EDS-RDC) 2013-2014. MPSMRM, MSP, and ICF International, Rockville, Maryland, USA.

[13] Arnoldussen, D., Maeyens, C., Assenmaker, P., Kalambayi, B. and Kitoko, C. (2014) Etude anthropologique sur les refus et résistances à la vaccination dans le Katanga. UNICEF, Lumumbashi.

[14] World Health Organization (2015) World Health Organization Vaccination Coverage Cluster Surveys: Reference Manual. WHO, Geneva.

[15] Ba Pouth, S.F., Kazambu, D., Delissaint, D. and Kobela, M. (2014) Immunization Coverage and Factors Associated with Drop-Out in Children 12 to 23 Months in Djoungolo-Cameroon Health District in 2012. The Pan African Medical Journal, $17,91$.

[16] Lakew, Y., Bekele, A. and Biadgilign, S. (2015) Factors Influencing Full Immunization Coverage among 12-23 Months of Age Children in Ethiopia: Evidence from the National Demographic and Health Survey in 2011. BMC Public Health, 15, 728. https://doi.org/10.1186/s12889-015-2078-6

[17] Nzioki, J.M., Ouma, J., Ombaka, J.H. and Onyango, R.O. (2017) Community Health Worker Interventions Are Key to Optimal Infant Immunization Coverage, Evidence from a Pretest-Posttest Experiment in Mwingi, Kenya. The Pan African Medical Journal, 28, 21. https://doi.org/10.11604/pamj.2017.28.21.11255

[18] Patel, A.R. and Nowalk, M.P. (2010) Expanding Immunization Coverage in Rural India: A Review of Evidence for the Role of Community Health Workers. Vaccine, 28, 604-613. https://doi.org/10.1016/j.vaccine.2009.10.108

[19] Sommanustweechai, A., Putthasri, W., New, M.L., Aung, S.T., Theint, M.M., Tangcharoensathien, V., et al. (2016) Community Health Worker in Hard-to-Reach Rural Areas of Myanmar: Filling Primary Health Care Service Gaps. Human Resources for Health, 14, 64. https://doi.org/10.1186/s12960-016-0161-4

[20] Rainey, J.J., Watkins, M., Ryman, T.K., Sandhu, P., Bo, A. and Banerjee, K. (2011) Reasons Related to Non-Vaccination and Under-Vaccination of Children in Low and Middle Income Countries: Findings from a Systematic Review of the Published Literature, 1999-2009. Vaccine, 29, 8215-8221. https://doi.org/10.1016/j.vaccine.2011.08.096

[21] Russo, G., Miglietta, A., Pezzotti, P., Biguioh, R.M., Bouting Mayaka, G., Sobze, M.S., et al. (2015) Vaccine Coverage and Determinants of Incomplete Vaccination in Children Aged 12-23 Months in Dschang, West Region, Cameroon: A Cross-Sectional Survey during a Polio Outbreak. BMC Public Health, 15, 630. https://doi.org/10.1186/s12889-015-2000-2

[22] Mutua, M.K., Kimani-Murage, E. and Ettarh, R.R. (2011) Childhood Vaccination in 
Informal Urban Settlements in Nairobi, Kenya: Who Gets Vaccinated? BMC Public Health, 11, 6. https://doi.org/10.1186/1471-2458-11-6

[23] Wiysonge, C.S., Uthman, O.A., Ndumbe, P.M. and Hussey, G.D. (2012) Individual and Contextual Factors Associated with Low Childhood Immunisation Coverage in Sub-Saharan Africa: A Multilevel Analysis. PLoS ONE, 7, e37905.

https://doi.org/10.1371/journal.pone.0037905

[24] Egondi, T., Oyolola, M., Mutua, M.K. and Elungata, P. (2015) Determinants of Immunization Inequality among Urban Poor Children: Evidence from Nairobi's Informal Settlements. International Journal for Equity in Health, 14, 24. https://doi.org/10.1186/s12939-015-0154-2

[25] Etana, B. and Deressa, W. (2012) Factors Associated with Complete Immunization Coverage in Children Aged 12 - 23 Months in Ambo Woreda, Central Ethiopia. BMC Public Health, 12, 566. https://doi.org/10.1186/1471-2458-12-566

[26] Fatiregun, A.A. and Okoro, A.O. (2012) Maternal Determinants of Complete Child Immunization among Children Aged 12 - 23 Months in a Southern District of Nigeria. Vaccine, 30, 730-736. https://doi.org/10.1016/j.vaccine.2011.11.082

[27] Anand, S. and Barnighausen, T. (2007) Health Workers and Vaccination Coverage in Developing Countries: An Econometric Analysis. The Lancet, 369, 1277-1285. https://doi.org/10.1016/S0140-6736(07)60599-6

[28] Hu, Y., Shen, L., Guo, J. and Xie, S. (2014) Public Health Workers and Vaccination Coverage in Eastern China: A Health Economic Analysis. International Journal of Environmental Research and Public Health, 11, 5555-5566.

https://doi.org/10.3390/ijerph110505555 